\title{
Percutaneous treatment of hallux valgus in children
}

\begin{abstract}
Fourty two patients with hallux valgus and bunion who underwent M.I.S. surgery, (Minimal Insicion Surgery) were studied retrospectively. $100 \%$ presented pain, the inter-metatarsal angle less than $15^{\circ}$. The technique employed has been, $1^{\circ}$ metatarsal distal osteotomy (Reverdin-Isham), abductor Hallux tenotomy, and phalange osteotomy (Akin), in all cases by M.I.S. Average age 13 years and average follow-up 5.5 years. Average time of hospitalization 7 hours. Analgesyc used ibuprofen. Patient was able to walk three hours after the surgery was done. The angles measured revealed a significant change compared to the preoperative time.
\end{abstract}

Complications: One infection, one recidivation. No pain. Parents satisfaction $100 \%$.

Keywords: Hallux valgus, Minimal incision surgery, Bunion, Children patients
Volume 8 Issue 6 - 2017

\author{
Mario Juanto \\ Universidad Nacional de Rosario, Argentina
}

Correspondence: Mario Juanto, Calle Dorrego No. 216, 2000,

Rosario,Argentina Email junatomario@hotmail.com

Received: August 04, 2017 | Published: August 23, 2017

\section{Introduction}

The treatment of bunion or hallux valgus dates from half of the twentieth century. Introduced by Reverdin and Riedel, it was then developed by several surgeons and it's being used nowadays, where we may find at least 130 operations for correcting it.

Women's feet are experiencing nowadays a series of changes, mainly because of new fashion trends like the use of pointed highheeled shoes, that lead to the deformity we are going to deal with. You could modify a quadrangular structure like the foot in a rhomboidal structure as a pointed shoe thanks to a series of predisposing factors, in addition to factors that trigger the lesions.

Even though hallux valgus is not a common pathology neither in teenagers nor in children, when the first symptoms appear it's essencial to treat it as fast as possible since it causes pain and functional changes. The surgical treatment has evolved over the years, and it has become less agressive. The most common surgical techniques for the correction of this deformity were distal osteotomy of the first metatarsal, Akin osteotomy, abductor osteotomy, and exostectomy as well.

After getting to know the MIS (Minimal Incision Surgery) performed on adults with hallux valgus, developed by Dr Stephen Isham, we were determined to put it into practice in pediatric patients since it's less aggressive compared to other techniques, and allows early return to normal activities.

The purpose of this article was to show the preliminary results by using the aforementioned technique with MIS.

\section{Materials and method}

From 2004 and 2009, we performed a retrospective study of forty two patients diagnosed with bilateral hallux vallux underwent MIS procedure. All of them presented pain, and the angle between the first and second metatarsal was less than $15^{\circ}$. All of them girls, the older 16 years and youngest 11 years

\section{Technique}

This treatment was performed with the patient in the supine position, leaving the foot out of the stretcher and using image intensifier.

Exostectomy: Firstly, we made a small incision of $2 \mathrm{~mm}$ on the internal face of the foot, behind the sesamoids, using a scalpel 64 (with the respective scalpel handle). Secondly, we perform the exostectomy with a drill Shannon 44 of $2 \mathrm{~mm}$ of lateral cut with an Osada Surgical Handpiece System (Figure 1). Then, we change the drill and insert a wedge burr of 3.1 or 4.1 to complete the exostectomy (2000-4000 $\mathrm{rpm})$. And finally, we remove the rest of bone by pressing the area, or with the DPR curette, made for that purpose.

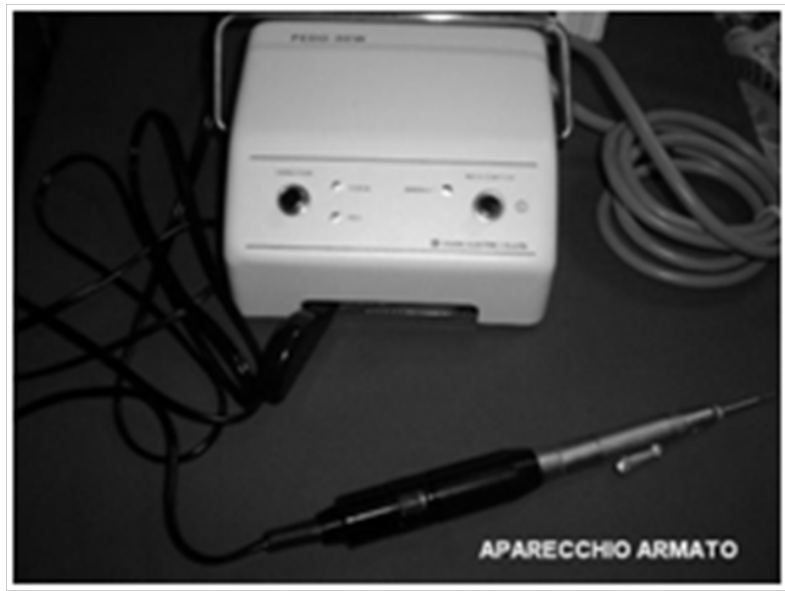

Figure I Osada Surgical Handpiece System.

Reverdin- Isham osteotomy: The distal osteotomy of the first metatarsal is performed through the same incision with a drill Shannon 44 , going from dorsal to plantar direction, and from distal to proximal.

Abductor tenotomy: Helped by an image intensifier we insert the scalpel at the metatarsophalangeal joint, and once it's inside we rotate it and making one only movement we section the lateral capsule and the abductor hallucis tendon.

Akin osteotomy: This procedure is done with a dorsal and internal incision, placed $1 \mathrm{~cm}$ distal to the MTCF articulation; then, we insert a drill Shannon 44 and we perform the osteotomy in order to correct the DASA (Distal Articular Surface Angle).

After these procedures are made we put on special bandage and postoperative footwear with a thick sole, thereby allowing a proper gait without displacing the osteotomy.

The first cleaning is made on the seventh postoperative day by putting a silicone toe separator for bunions, as well as dressing, with a coban bandage of $10 \mathrm{~cm}$. 
Patients were assessed with a clinical and radiographic protocol with a follow-up of 5 years (the oldest) and 9 months (the youngest).

\section{Results}

The overall time of hospitalization was 7 hours approximately. The analgesyc used to relieve the pain was ibuprofen in the early postoperative period (48 hours), and the patient was able to walk three hours after the surgery was done with an special shoe. The parents of the $100 \%$ of the patients were satisfied of the result either cosmetic or functional.

The metatarsophalangeal angle, PASA (Proximal Articular Surface Angle), and DASA, were measured radiographically and revealed a significant change compared to the preoperative time. The metatarsophalangeal joint improved from a preoperative mean value of $18^{\circ}$ to a postoperative of $6^{\circ}$. The PASA showed a mean preoperative value of $12^{\circ}$ and was improved to $4^{\circ}$. And the DASA progressed from a mean preoperative of $8^{\circ}$ to a postoperative of $2^{\circ}$ (Figure 2-4).

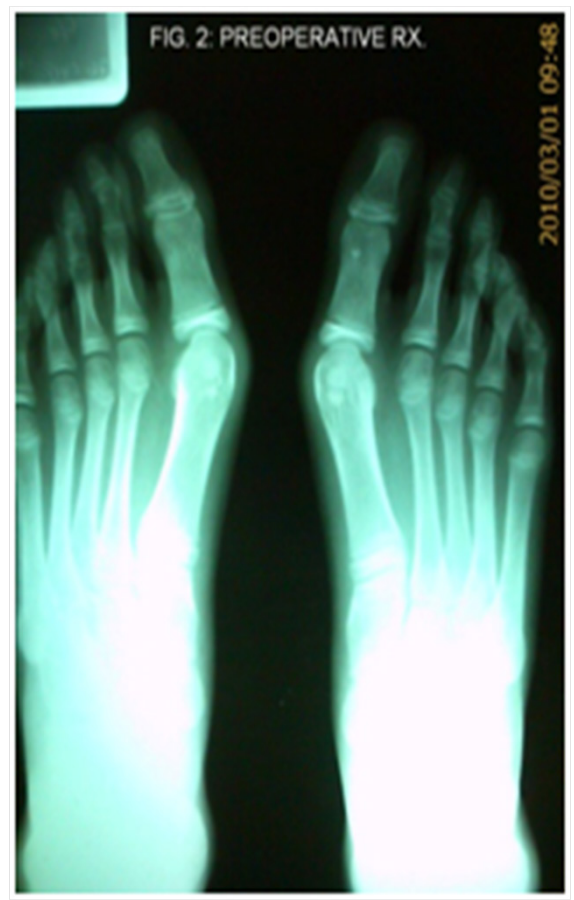

Figure 2 Preoperative $\mathrm{RX}$.

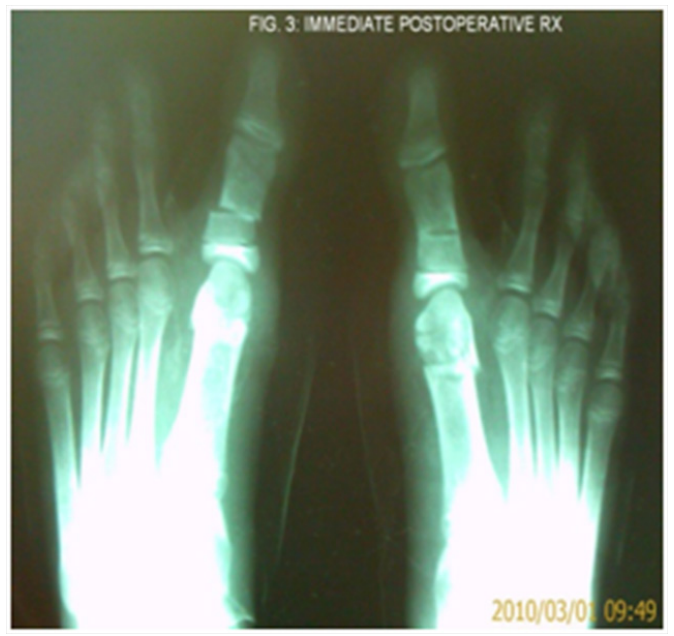

Figure 3 Immediate Postoperative RX.

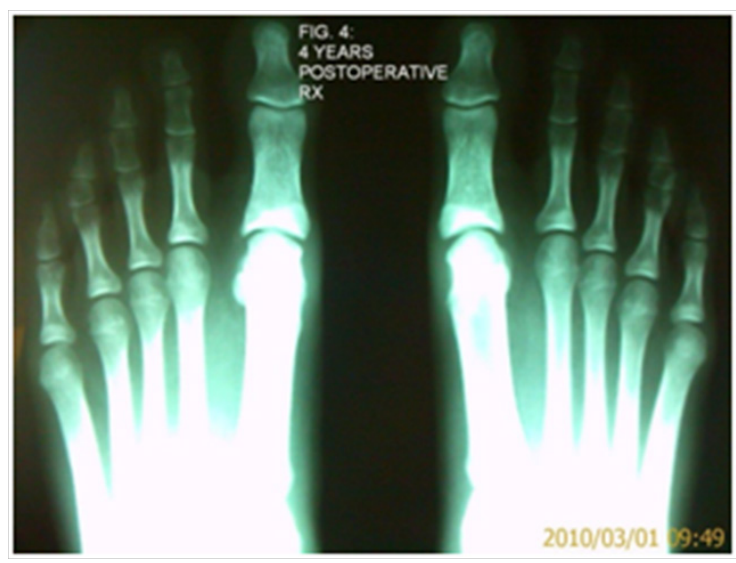

Figure 4 Four years postoperative RX.

There was only one problem reported in the cases with a 12-yearold girl involving an infection of one of the Reverdim-Isham osteotomy incision, that improved with antibiotic treatment as well as resting. There was no recidivation diagnosed with any of the feet at the metatarsophalangeal angle to date, and we felt compelled to perform the procedure again; no patients suffered from pain during the evaluation.

\section{Discussion}

As regards studies on MIS in adult patients there were published only a few, whereas in child patients there were presented no cases. As for open surgeries both in children and in teenagers, we have only found several articles within the literature review. ${ }^{1-10}$

In our hospital, we used to carry out the Mitchel osteotomy - 3 or 4 years ago- with positive postoperative results, but we were forced to stop using that technique since it was difficult to operate through it and the postoperative pain was important because of the open surgery. $2,5,6,8$

This procedure was performed in children by Dr. Quintan Rodriguez J. together with his co-workers from Hospital La Paz, Madrid. In their book "Homagge to Dr. Bernardo Esteban Mujica" they named it Pelet osteotomy -pages 203 to 209- that was done adding soft-tissue technique described by Viladot.

Chevron osteotomy was another open technique that we used regularly, and it prolonged the postoperative period. We had to perform it with an osteosynthesis with screw in order to keep stability, turning it into a more-expensive procedure -which is a big problem in our country.

This osteotomy could also be performed with MIS, as orthopedist Mariano del Prado, from Murcia, describes in his book "Cirugía Percutánea del Pie" (Percutaneous Technique of the Foot) belonging to the Spaniard publishing house Masson.

In the article "Juvenile Hallux Valgus: Etiology and Treatment", by Coughlin M J, 45 patients (60 feet) were evaluated. Half of them were treated with Chevron osteotomy, and the rest with McBride procedure. Patients that showed most severe manifestations were treated with distal and proximal double osteotomy combined with soft-tissue techniques. Good and excellent results were obtained in $92 \%$ of cases following this procedure.

Several physicians are focused on minimally invasive techniques, but as many authors propose we should apply in adult patients the osteotomy developed by Bosh in the 80 's. This procedure consists of metatarsal neck osteotomy that corrects the PASA (Proximal 
Articulation Surface Angle), adding a lateral displacement of the head and it results in the correction of the intermetatarsal angle ( $1^{\mathrm{a}}$ IMTT). In our series the $1^{\text {a }}$ IMTT, was below the $12^{\circ}$.

Results published are similar to those of Dr. Migues (Hospital Italiano, Capital Federal) and Dr. Nicolla Maffulli (Department of Orthopaedic, University Clinic Hospital Keele, England), suggesting that good an excellent results were near the $95 \%$ in 15 adult patients (21 feet).

Groisso. ${ }^{11}$ published in the Journal of Pediatric Orthopaedic his article "Juvenile hallux valgus: A Conservative Approach to Treatment"; he used a conservative method consisting of use of a thermoplastic splint, as well as passive and active exercises for the treatment of hallux valgus in child-teenager patients. Later results suggest the success of his work, but were subordinated to a personal and longer treatment, help of the parents, and frequent controls.

Orthopedist Schwitalle M and his colleagues from Orthopaedic University Clinic Hospital Mainz, Germany, published their article "Hallux Valgus in Young Patients: Comparison of Softtissue Realignment and Metatarsal Osteotomy". They carried out a comparative research in which two open techniques of hallux valgus correction in young patients were compared by long-term results: soft-tissue realignment (McBride) and Mitchell osteotomy. McBride procedure showed $75 \%$ of good and excellent results, whereas outcomes after Mitchell osteotomy were graded in $85 \%$.

\section{Conclusion}

As we know this is a preliminary study, but we are very happy because the results suggest similarity with open-surgery methods, with the additional advantage of less time of surgery, less time of hospitalization, less pain and good cosmetic results.

\section{Acknowledgments}

None.

\section{Conflicts of interest}

None.

\section{References}

1. Dabbah F, Dabbah G Evaluación alejada del trasplante del abductor del hallux en el tratamiento del hallux valgus con pie plano transverso. Nuestra experiencia en el tratamiento del hallux valgus con pie plano transverso según técnica quirúrgica de la escuela del Hospital Rawson. Rev Asoc Arg Ortop y Traumatol 1994;59(2):171-181.

2. J Quintan Rodríguez, Y Cols Libro Homenaje al Dr. Bernardo Esteban Mujica, pp. 1995;203-209.

3. Albrecht E Pathology and treatment of hallux valgus. Russki Vrach. 1911;10:14.

4. Campbell Crenshaw Lean Manufacturing in the Developing World. (7a edn). 1988

5. Durman Dc Metatarsus primus varus and hallux valgus. AMA Arch Surg. $1957 ; 74(1): 128$

6. Kelikian H Hallux valgus, allied deformities of the forefoot and metatarsalgia. Philadelphia, USA. 1965

7. Johnson AE Treatment of adolescent hallux valgus with the first metatarsal double osteotomy: the denver experience. J Pediatr Orthop. 2004;24(4):358-362.

8. Schwitalle M Hallux valgus in young patients: comparison of softtissue realignment and metatarsal osteotomy. Eur J Pediatr Surg. 1998;8(1):42-46.

9. Senaris-Rodriguez J Surgical treatment for bunions in adolescents. $J$ Pediatr Orthop. 1998;7(3):210-216.

10. Magnan B Percutaneous distal metatarsal osteotomy for correction of hallux valgus. Surgical technique J Bone Joint Surg Am 88 Suppl 1 Pt. 2006;1:135-148.

11. Groisso JA Juvenile hallux valgus. A conservative approach to treatment J Bone Joint Surg Am. 1992;74(9):1367-74. 